\title{
Microglia polarization in heat-induced early neural injury
}

Lei Wang ${ }^{1}$, Jing Zhao ${ }^{2}$, Baofeng Zhu ${ }^{1}$, Junhua Shen ${ }^{1}$, Zi Ye ${ }^{3}$, Qiang Peng ${ }^{1}$, Yi Zhang ${ }^{3}$

\author{
${ }^{1}$ Department of Emergency Center, Affiliated Hospital 2 of Nantong University, Nantong, \\ Jiangsu, China \\ 2Department of Dermatology, Affiliated Hospital 3 of Nantong University, Nantong, Jiangsu, \\ China \\ ${ }^{3}$ Department of Neurosurgery, Affiliated Hospital 2 of Nantong University, Nantong, Jiangsu, \\ China
}

Submitted: 23 September 2019; Accepted: 12 January 2020

Online publication: 11 August 2022

Arch Med Sci

DOI: https://doi.org/10.5114/aoms/116550

Copyright $\odot 2021$ Termedia \& Banach

\section{Abstract}

Introduction: In the occurrence and development of heat stroke (HS), factors such as hyperthermia, ischemia and hypoxia are essential to the central nervous system (CNS) inflammatory response, but the main mechanism underlying CNS inflammation remains unclear. The aim of the study was to observe the polarization of microglia in response to heat-induced early nerve injury and to explore its possible mechanism of action.

Material and methods: To establish a heatstroke animal model in Beagle dogs, 18 Beagle dogs were divided into control (group A) and experimental groups (group B, group $C$ and group D) according to a random numbers table. The animals in the experimental groups were placed on an electric blanket of an animal body temperature maintaining apparatus. The temperature was set at $40 \pm 0.5^{\circ} \mathrm{C}$, and the rectal temperature was monitored every 5 min until the target body temperature was reached. Once the target temperature was reached, the dogs were transferred to an environment of 26 $\pm 0.5^{\circ} \mathrm{C}$ and $60 \pm 0.5 \%$ humidity. Western blot analysis was used to detect the expression of microglia-specific markers CD45, iNOS, arginase, and CD206 in normal and heat-damaged brain tissues at different time points ( $1 \mathrm{~h}$, $6 \mathrm{~h}, 24 \mathrm{~h}$ ). The expression of CD45 and arginase was further determined by co-localization with immunofluorescence.

Results: CD45 and iNOS protein expression was detected in group A. The two protein markers in group $B$ were significantly higher than those in group $A(p<0.05)$, and the protein markers in group $C$ were still higher than those in group A $(p<0.05)$. There was no statistically significant difference among the animals in group A $(p>0.05)$. Arginase and CD206 protein expression was also detected in group $A$. Levels of the two protein markers in group $B$ were higher than those in group A $(p<0.05)$, and the protein marker levels in group $C$ were even higher than those in group $A(p<0.05)$. Further analysis of the two groups of protein markers in group $D$ showed significantly higher levels than those in group A $(p<0.001)$. Immunofluorescence co-localization of CD45 and arginase showed significantly increased fluorescence density at $6 \mathrm{~h}$ and $24 \mathrm{~h}$ after thermal injury $(p<0.001)$.

Conclusions: After heat-induced disease, microglia were found to be active in the brain tissues of dogs. The microglia activated in the early 1-6 h of CNS injury were mainly the $\mathrm{M} 1$ type, which were then converted to the $\mathrm{M} 2$ type after $6 \mathrm{~h}$. The $24 \mathrm{~h} \mathrm{M} 2$ type was dominant. The relationship between M1/M2 polarization trends and early brain injury in heat-induced disease may be a key to understanding CNS injury in heat-induced disease.

Key words: microglia, polarization, heat stroke, brain injury, Beagle dogs.

\author{
Corresponding author: \\ Yi Zhang MD \\ Department of Neurosurgery \\ Affiliated Hospital 2 \\ of Nantong University \\ No. 6 Hai Er Xiang Bei Lu \\ Nantong, Jiangsu, China \\ Phone: 86051385061012 \\ Fax: 86051385061003 \\ E-mail: \\ zhangyi9285@sina.com
}




\section{Introduction}

In the occurrence and development of heat stroke (HS), factors such as hyperthermia, ischemia and hypoxia are essential to the central nervous system (CNS) inflammatory response, but the main mechanism underlying CNS inflammation remains unclear [1]. Microglia are persistent sensors of microenvironmental changes in the CNS and restorers of tissue homeostasis. Along with their role as the main immune cells of the CNS, they regulate the innate immune function of other glial cells. Neuroinflammation induces the activation of molecular isomerically reactive microglia that produce deleterious (M1-type) or beneficial (M2-type) responses [2, 3]. Microglia in the healthy adult CNS are characterized by a quiescent morphology. After they acquire an activated, amoeboid morphology in response to invading pathogens and/or CNS damage they increase their production of a wide array of cytokines, reactive oxygen species and inducible nitric oxide synthase (iNOS) that induce neuroinflammation $[4,5]$. At the site of CNS injuries, macrophages are derived from both microglia and hematogenous macrophages. The presence of M1/M2 phenotypic polarization has been suggested for microglia, which have been linked to functional properties including production of inflammation association molecules and phagocytic activity. Recent studies show that microglia can be polarized to a 'classically' activated M1 cell that is pro-inflammatory and cytotoxic, or to an 'alternatively' activated $\mathrm{M} 2$ cell that is anti-inflammatory and has reparative properties [6]. Microglia likely undergo phenotypic changes during ontogenesis, although in the healthy brain they did not exclusively express either M1 or M2 phenotypic markers at any time [7]. Studies have shown that the typical antigen markers of activated M1 microglial include CD45, CD86, CD64 and iNOS, and the microglia express high levels of the M2 markers CD206 (mannose receptor), CD163 (hemoglobin-haptoglobin scavenger receptor) and arginase 1 (Arg1) [6].

This study aimed to investigate the molecular mechanism of CNS inflammation involved in thermal injury by establishing a Beagle canine heatstroke model to analyze the expression of different types of microglia induced by thermal injury stimulation and to determine whether it could affect microglial polarization.

\section{Material and methods}

\section{Experimental subjects and grouping}

Eighteen normal grade 6-month-old Beagle dogs (male) with weights of $15 \pm 0.5 \mathrm{~kg}$ were provided by the Animal Experimental Center of Nan- tong University Medical College. The animal experiments in this study were approved by the ethics committee of Nantong University Medical College (Ethics council approved number: SCXK (Su): 20080010). Animals were kept separate from each other to ensure proper temperature for raising. All experimental procedures involving animals were in accordance with the guidelines for the use of experimental animals established by the National Health Association, and met the requirements for animal use and operation of the Animal Experimental Center of Nantong University.

All adult Beagle dogs were randomly divided into four groups. Nine animals were arranged in the normal control group (group A). The remaining animals were divided into the $1 \mathrm{~h}$ group of heat-radiation disease (group B), the $6 \mathrm{~h}$ group of heat-radiation disease according to the exposure time after thermal injury (group C), and the 24-h group of heat-shooting disease (group D), with three experimental animals in each sub-group. The animals in different heat-radiation groups were sacrificed and their hypothalamus was frozen or processed for primary microglia culture.

\section{Main instruments and reagents}

Animal temperature maintenance instrument XR200 (Xinsoft Information Technology, Shanghai) body temperature measurement setting range -30 to $60^{\circ} \mathrm{C}$. Animal Blood Pressure Data Collection and Analysis System BP-2000 (Yawentai, Beijing). Fluorescence microscope (DP 71, Olympus, Japan).

Radioimmunoprecipitation test lysate (Biyuntian Bio, Shanghai); phenylmethylsulfonyl fluoride (subfamily, Suzhou); BCA protein concentration determination kit (Biyuntian Bio, Shanghai); internal reference $\beta$-actin (Ai Meijie, Wuhan); primary antibody monoclonal antibody CD45 (Aibo, Shanghai), rabbit polyclonal antibody iNOS (Aibo, Shanghai); rabbit polyclonal antibody arginase (Aibo, UK), rabbit polyclonal antibody CD206 (Amy Jie, Wuhan); phosphate buffered saline solution (Biyuntian Bio, Shanghai); secondary anti-goat anti-mouse IgG $(\mathrm{H}+\mathrm{L})$ Alexa Fluor Plus 488 (Thermo Fisher, Shanghai).

\section{Experimental method}

\section{Beagle canine heat shock injury model}

Diagnostic criteria for experimental heat-radiation disease referring to the diagnostic criteria for animal heat-radiation disease are as follows [8]: 1) core body temperature reaches $40^{\circ} \mathrm{C}$ with symptoms of nervous system damage such as prospecting, convulsions, and coma; 2) core temperature reaches $42.5^{\circ} \mathrm{C}$ or artery shows a point of decline after which the systolic blood pressure 
rises to a peak indicating that moderate heatstroke has occurred; 3) a severe heatstroke is considered when the core temperature reaches $43.0^{\circ} \mathrm{C}$ or the arterial systolic pressure drops below $90 \mathrm{~mm} \mathrm{Hg}$.

All Beagle dogs were intravenously anesthetized with $3 \%$ sodium pentobarbital ( $1 \mathrm{ml} / \mathrm{kg}$ ) for $12 \mathrm{~h}$ before fasting. When the corneal reflex and pain reflex both disappeared, and the breathing and blood pressure were stable, then the anesthesia was considered satisfactory. In the control group, nine Beagle dogs were placed under an environment of $26 \pm 0.5^{\circ} \mathrm{C}$ until the end of the experiment without any other treatment. On the electric blanket of the animal temperature maintenance instrument was the place where the nine experimental group animals were arranged, and the temperature was set at 40 $\pm 0.5^{\circ} \mathrm{C}$, and the core body temperature (rectal temperature) was monitored every $5 \mathrm{~min}$. When the core body temperature reached $40^{\circ} \mathrm{C}$, the observation continued, while the average arterial pressure measurements were initiated. Falling of the arterial pressure as a time node is considered successful for thermal imaging modeling. After successful modeling, all experimental animals were transferred to an environment of 26 $\pm 0.5^{\circ} \mathrm{C}$ and $60 \pm 0.5 \%$ humidity, and routinely bred, with ECG and hemodynamic monitoring performed simultaneously.

As shown in Figure $1 \mathrm{~A}$ and $1 \mathrm{~B}$, the Beagle dogs were placed on the operating table for $1 \mathrm{~h}, 6 \mathrm{~h}$, and $24 \mathrm{~h}$ with the head fixed in a lateral position, the ear edge fixed to the caudal side, and anesthesia was administered. The craniotomy was performed through a sacral approach. After finishing craniotomy, the subcutaneous tissue was turned to the lateral bone flap, and the bone window was as close as possible to the skull base. After washing off excess blood with physiological saline, brain tissue was placed in a tube and stored at $-80^{\circ} \mathrm{C}$ for later use. Next, $100 \mathrm{mg}$ of brain tissue was used to prepare $1 \mathrm{ml}$ of cell lysate, homogenized, centrifuged, and the supernatant aspirated. After adding a portion of the protein buffer and boiling for $15 \mathrm{~min}$, western blot was performed. The supernatant of the remaining cells was placed at $-80^{\circ} \mathrm{C}$ for future use.

\section{Western blot analysis of microglia-specific protein marker expression}

The brain tissues of the normal group and different time points after thermal injury were examined by western blot, and the expression of CD45, iNOS, arginase, and CD206 in the 4 groups of microglia was observed. Protein was quantified by the BCA assay, separated by polyacrylamide gel electrophoresis, and then transferred to
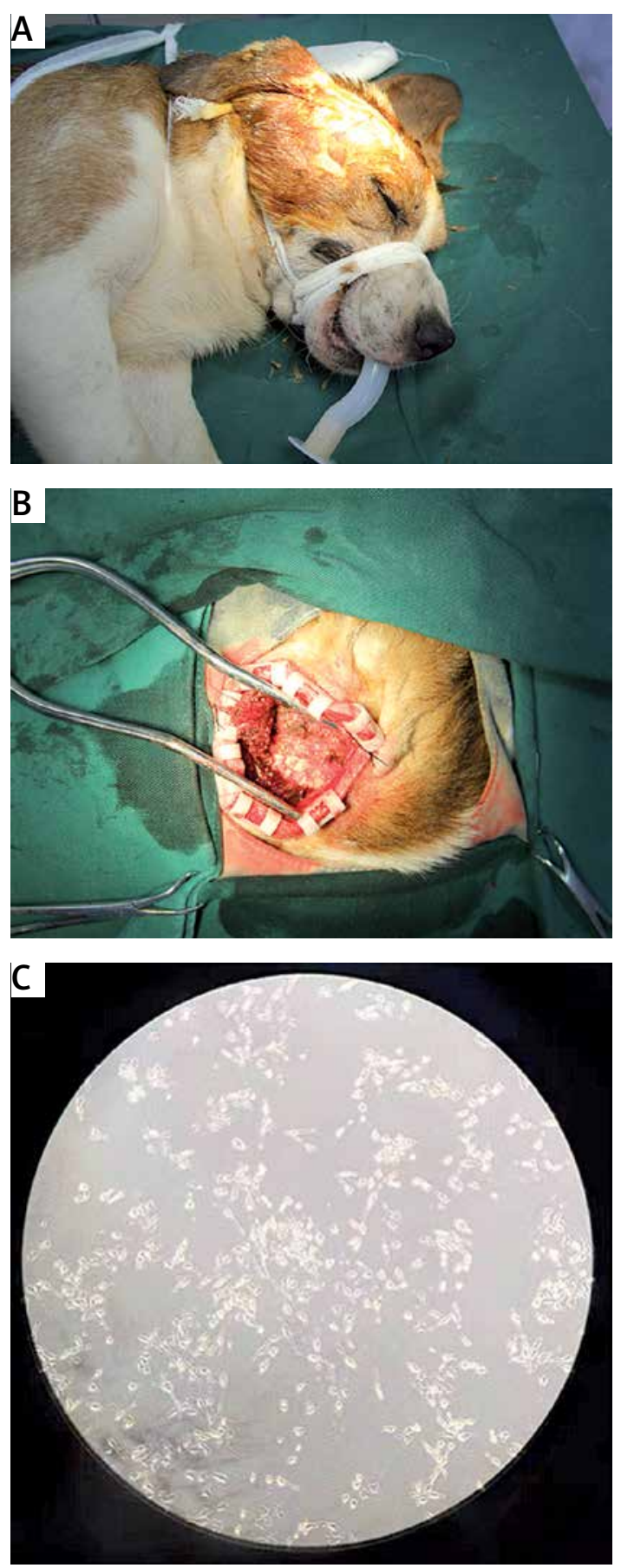

Figure 1. Beagle canine heat shock injury model. A - The Beagle dog is placed in the lateral position, the head is rotated to the opposite side, and the ear edge is pulled toward the midline and the caudal side. B - Craniotomy was performed through a sacral approach. After the craniotomy was fully performed, the subcutaneous tissue was turned to the lateral bone flap, and the bone window was as close as possible to the skull base. C - Primary microglia (100 × magnification)

a polyvinylidene fluoride membrane, followed by blocking with $5 \%$ Tris- $\mathrm{Cl}$ (pH 6.8) containing 5\% skim milk powder at room temperature for $1 \mathrm{~h}$. After TBST rinse, antibodies against CD45, iNOS, 
A

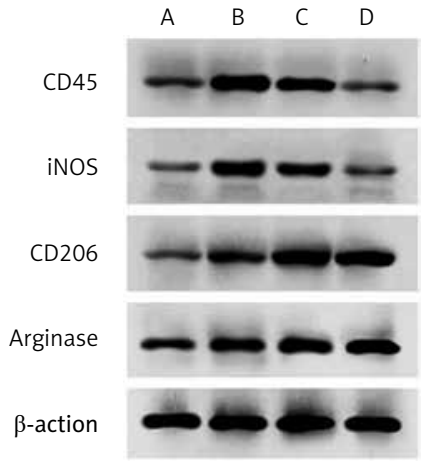

B

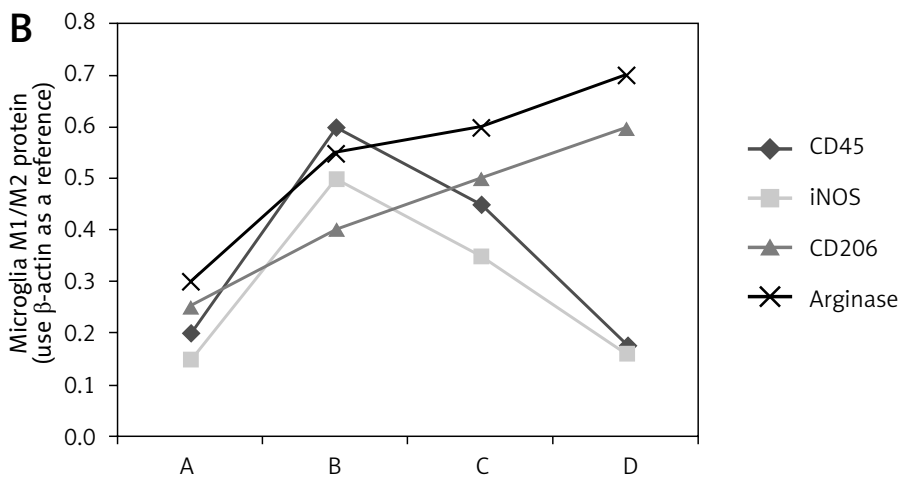

Figure 2. M1/M2 protein expression of microglia after heat injury in each group. A - is the normal control group; $\mathrm{B}$ - is the $1 \mathrm{~h}$ group after thermal injury; C - is $6 \mathrm{~h}$ group; D - is $24 \mathrm{~h}$ group. CD 45 - leukocyte differentiation antigen 45, iNOS - inducible nitric oxide synthase, CD206 - leukocyte differentiation antigen 206. A - The expression of M1/M2 specific protein in microglia by western blot after thermal injury in Beagle dogs. $\mathbf{B}-$ The results of corresponding western blot analysis

arginase, CD206 and $\beta$-actin antibody were incubated overnight at $4^{\circ} \mathrm{C}$, rinsed 5 times with TBST for $5 \mathrm{~min}$, and then incubated with HRP-labeled secondary antibody for $2 \mathrm{~h}$ at room temperature. Following rinses 3 times with TBST for $15 \mathrm{~min}$, chemiluminescent substrate was added followed by dark room exposure and development. Strip gray values were determined using Image J analysis.

\section{Immunofluorescence analysis}

The hypothalamic tissues from three groups were trypsinized to extract the primary microglia and cell morphology was observed under an electron microscope (Figure $1 \mathrm{C}$ ), then cells were transferred to a Petri dish and cultured with an appropriate amount of medium. Immunofluorescence analysis was used to detect the expression of CD45 cells and arginase immunoreactive cells after heat exposure stimulation. The stabilized nerve cells were cultured with medium discarded. Then, cells were rinsed with PBS, fixed in $4 \%$ paraformaldehyde for 40 min at room temperature, rinsed 3 times with PBS for $10 \mathrm{~min}$, and then incubated with fluorescent blocking solution for $2 \mathrm{~h}$ at room temperature. After completion, CD45 antibody (microglia M1 type), arginase antibody (microglia M2 type) was incubated overnight at $4^{\circ} \mathrm{C}$, rinsed 3 times with PBS for 5 min, and stained with Dylight 594 $(1: 400)$ and Dylight $488(1: 400)$ fluorescent labels. The secondary antibody was incubated at room temperature for $2 \mathrm{~h}$ in the dark, and rinsed another 3 times with PBS for 5 min, DAPI counterstain was added for 30 min in the dark, then it was rinsed again 3 times with PBS for $15 \mathrm{~min}$, and then sealed with fluorescent sealing liquid. Cells were observed and photographed using a confocal microscope. Fusion image overlay showing double-marked cells was obtained.

\section{Statistical analysis}

The results of the experiment were statistically analyzed using SPSS 19.0 software. All data were expressed as mean \pm standard deviation $(\bar{x} \pm s)$, and CD45 and arginase results were compared by one-way ANOVA. The difference was statistically significant at $p<0.05$.

\section{Results}

\section{CD45 and iNOS protein expression in Beagle dogs}

The M1 type specific marker proteins CD45 and iNOS were detected by western blot in the hypothalamus of experimental animals from the four groups. A group of CD45 and iNOS proteins was detected in group $A$. The levels of the two protein markers in group $B$ were significantly higher than those in group $A(p<0.05)$, and the protein marker levels in group $C$ were still higher than those in group A $(p<0.05)$. There was no significant difference among the animals in group $A(p>0.05)$. It was suggested that the expression of CD45 and iNOS was increased significantly at $1 \mathrm{~h}$ after thermal injury, gradually decreased after $6 \mathrm{~h}$ and returned to the normal control level after $24 \mathrm{~h}$ (Figure $2 \mathrm{~A}$ and $2 \mathrm{~B}$ ).

\section{Arginase and CD206 protein expression in Beagle dogs}

M2 type specific markers arginase and CD206 were detected in the brain tissue of experimental animals from four groups. A group of arginase and CD206 proteins was detected in group $A$. The levels of the two protein markers in group $B$ were much higher than those in group $A(p<0.05)$, and the protein marker levels in group $C$ were even higher than those in group A $(p<0.05)$. Further analysis was then performed. Levels of both pro- 

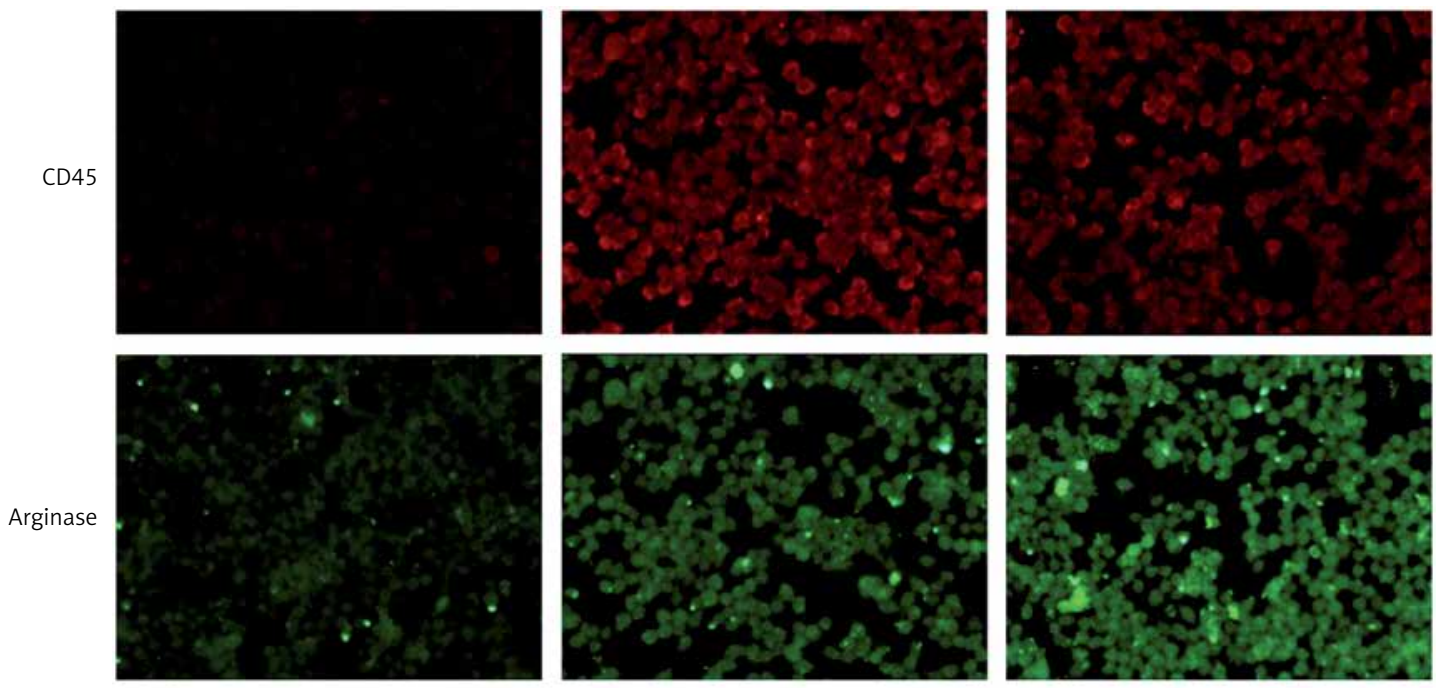

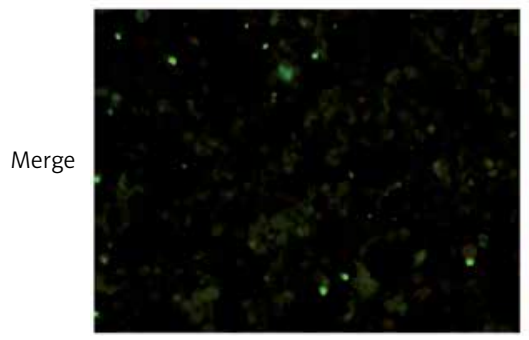

$1 \mathrm{~h}$

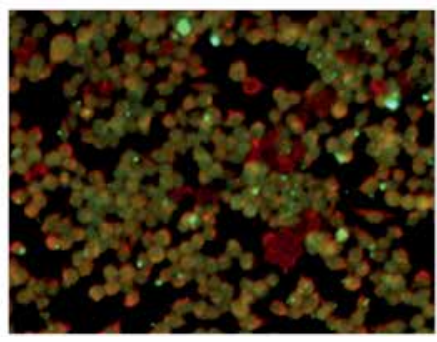

$6 \mathrm{~h}$

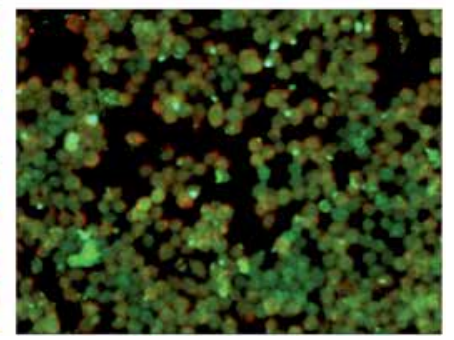

$24 \mathrm{~h}$

Figure 3. Immunofluorescence co-localization of M1/M2 marker expression (bar $=100 \mu \mathrm{m}$ ). CD45 showed a red fluorescent label and arginase showed a green fluorescent label (×400, magnification)

tein markers were significantly higher in group D than in group $\mathrm{A}(p<0.001)$. It was thus suggested that the expression of arginase and CD206 proteins began to increase at $1 \mathrm{~h}$ after thermal injury and reached a peak after $24 \mathrm{~h}$ (Figure $2 \mathrm{~A}$ and $2 \mathrm{~B}$ ).

\section{Immunofluorescence co-localization of $\mathrm{M} 1 / \mathrm{M} 2$ marker expression}

As shown by immunofluorescence, CD45 showed a red fluorescent label and arginase showed a green fluorescent label. CD45 and arginase showed low expression in the normal control group. We observed the immunofluorescence of each subgroup after heat shock: thermal injury for $1 \mathrm{~h}$ (group B), $6 \mathrm{~h}$ (group C), and $24 \mathrm{~h}$ (group D). A small amount of red fluorescence was observed in group $B$ and group $D$, and a significant amount of red fluorescence was observed in group $C$, which was different from group $B$ and group D $(p<0.001)$, suggesting that the expression of CD45 was increased after $6 \mathrm{~h}$ of thermal injury. In addition, the green fluorescence of group $\mathrm{C}$ was higher than that of group B $(p<0.001)$, while that of group $D$ was the most obvious $(p<0.001)$. It suggested that the expression of arginase increased $6 \mathrm{~h}$ after heat injury, and the most obvious increase was $24 \mathrm{~h}$ after heat injury (Figure 3).

\section{Discussion}

Heat stroke is a serious clinical symptom caused by elevated core temperature, from which patients will suffer from varying degrees of CNS damage. The vulnerable areas in the CNS are mainly in the hypothalamus, cerebellum, and hippocampus [9]. Related studies have indicated that hyperthermia and neuroinflammatory reactions are the main causes of central brain injury in HS patients. Although early active treatment can be achieved to save the lives of most HS patients, one-third of these patients will display permanent nerve damage [10]. At the early stage of HS, cerebral edema will occur, which is mostly reversible compared with irreversible cerebral edema caused by trauma and cerebral hemorrhage and embolism [11]. Once stimulated by various pathological stimuli, microglia in the CNS will be fleetly activated. Afterwards, microglia will mainly manifest two phenotypes: M1 type microglia that mainly secrete pro-inflammatory factors such as $\mathrm{IL}-1 \beta$, IL-6, TNF- $\alpha$, and NO, exerting neurotoxic effects including promoting inflammation and inhibiting phagocytosis; and M2 type microglia that mainly secrete anti-inflammatory factors, transforming growth factor beta (TGF- $\beta$ ), arginase, and neurotrophic factors with neuroprotective effects including regeneration. The relationship between 
M1/M2 polarization trends and early brain injury in heat-induced disease is the key to CNS injury in thermal radiation.

Previously, mice were mostly used as the thermal injection model [12-14]. However, in the present study, Beagle dogs were chosen to be the model subjects, considering that large animals such as dogs can be installed with ECG monitoring and hemodynamic monitoring to obtain real-time data. Moreover, more pathophysiological data can be acquired after changing the environmental setting parameters and dynamically observing the animal activity parameters. The expression of M-type polarizing markers CD45 and iNOS in 1-6 h microglia reached a peak at $6 \mathrm{~h}$, and gradually decreased during the recovery time, then returned to normal at $24 \mathrm{~h}$. The expression levels of M2-type polarized markers arginase and CD206 began to increase gradually from 1 to $6 \mathrm{~h}$ after $\mathrm{HS}$, and reached the highest level after $24 \mathrm{~h}$. Furthermore, the expression of $M 1$ and $M 2$ activated phenotype molecules in microglia was observed by immunofluorescence-labeled laser confocal microscopy. The M1 activation marker was marked at $6 \mathrm{~h}$ after heat shock, but it was significantly weakened after $24 \mathrm{~h}$. The $\mathrm{M} 2$ activation marker became active $6 \mathrm{~h}$ after $\mathrm{HS}$ and was clearly marked at $24 \mathrm{~h}$. The results indicated that $M 1$ type microglia were mainly activated in the early $1-6 \mathrm{~h}$ of CNS injury and $M 2$ type microglia were mainly activated after 6-24 h, M2 being dominant at $24 \mathrm{~h}$. Biedenkapp et al. [15] established a mouse thermal radiation model to observe the changes in gene expression of various cytokines and chemical factors in a recovery period within $24 \mathrm{~h}$, and found that the expression of inflammation-related factor genes began to increase significantly within $2 \mathrm{~h}$ after heat-induced disease and returned to the control level at $24 \mathrm{~h}$, which was similar to our experimental results. In this experiment, we chose $1 \mathrm{~h}, 6 \mathrm{~h}$, and $24 \mathrm{~h}$ as the phase observation points. In the future, we will continue to expand the animal sample size and observe the dynamic changes in the central nervous microglia in an hourly manner.

Studies have shown that pre-heavy and light, with gradual recovery are the main characteristics of the CNS inflammatory response and $\mathrm{HE}$ histopathological changes within $24 \mathrm{~h}$ after thermal injury [16]. This trend is in line with the M1/ M2 polarization trend of microglia. At the early stage of heat radiation, small glial cells gradually become polarized to $M 2$ type, and their antiinflammatory and prosthetic effects result in heat-induced disease. The CNS inflammatory response and histopathological damage are gradually alleviated and restored. Therefore, microglia may be one of the important targets of thermal stimulation-mediated CNS injury, and regulating their polarization by limiting $\mathrm{M} 1$ or promoting $\mathrm{M} 2$ activation may become a potential strategy for the treatment of brain damage caused by heat-induced diseases.

In CNS diseases such as Parkinson's disease, Alzheimer's disease, atrophic lateral sclerosis, multiple sclerosis, and stroke, microglia can rapidly develop an early inflammatory reaction, followed by an astrocyte reaction [17-20]. Molecular dialogue among glial cells provides an important and novel direction for the CNS to participate in health and disease $[21,22]$. In a new era of glial cell biology, future research is needed to expand our understanding of the importance of crosstalk between resident and infiltrating immune cells in neurons, astrocytes, and the CNS [23]. Therefore, whether astrocytes play a role in the CNS inflammatory response of heat-induced disease is a point worthy of further study.

\section{Acknowledgements}

Lei Wang and Jing Zhao contributed equally to this work.

This research was supported by the 'Top Six Types of Talents' Financial Assistance of Jiangsu Province Grant (2019-WSW-199); the Nantong Science and Technology Project (JC2018088, JCZ18004). Nantong University School-level Fund Project (2019JZ005, 2019JY005).

\section{Conflict of interest}

The authors declare no conflict of interest.

\section{References}

1. Knapik JJ, Epstein Y. Exertional heat stroke: pathophysiology, epidemiology, diagnosis, treatment, and prevention. J Spec Oper Med 2019; 19: 108-16.

2. Baud O, Saint-Faust M. Neuroinflammation in the developing brain: risk factors, involvement of microglial cells, and implication for early anesthesia. Anesth Analg 2019; 128: 718-25.

3. Pandey MK, Sung B, Ahn KS, Kunnumakkara AB, Chaturvedi MM, Aggarwal BB. Gambogic acid, a novel ligand for transferrin receptor, potentiates TNF-induced apoptosis through modulation of the nuclear factorkappaB signaling pathway. Blood 2007; 110: 3517-25.

4. Graeber MB, Li W, Rodriguez ML. Role of microglia in CNS inflammation. FEBS Lett 2011; 585: 3798-805.

5. Streit WJ, Conde JR, Fendrick SE, Flanary BE, Mariani CL. Role of microglia in the central nervous system's immune response. Neurol Res 2005; 27: 685-91.

6. David S, Kroner A. Repertoire of microglial and macrophage responses after spinal cord injury. Nat Rev Neurosci 2011; 12: 388.

7. Crain JM, Nikodemova M, Watters JJ. Microglia express distinct $\mathrm{M} 1$ and $\mathrm{M} 2$ phenotypic markers in the postnatal and adult central nervous system in male and female mice. J Neurosci Res 2013; 91: 1143-51.

8. Lin $\mathrm{X}$, Lin $\mathrm{CH}$, Zhao T, et al. Quercetin protects against heat stroke-induced myocardial injury in male rats: anti- 
oxidative and antiinflammatory mechanisms. Chem Biol Interact 2017; 265: 47-54.

9. Chang CY, Chen JY, Chen SH, Cheng TJ, Lin MT, Hu ML Therapeutic treatment with ascorbate rescues mice from heat stroke-induced death by attenuating systemic inflammatory response and hypothalamic neuronal damage. Free Radic Biol Med 2016; 93: 84-93.

10. Higareda-Basilio AE, Trujillo-Narvaez FA, Jaramillo-Ramirez HJ. Mortality and functional disability in heat stroke. Salud Publica Mex 2019; 61: 99-100.

11. Du Y, Xu JT, Jin HN, et al. Increased cerebral expressions of MMPs, CLDN5, OCLN, ZO1 and AQPs are associated with brain edema following fatal heat stroke. Sci Rep 2017; 7: 1691.

12. Liu J, Wan M, Zhang Y, Zhang S, Zhang H, Wu S. Dysfunction of iron metabolism and iron-regulatory proteins in the rat hippocampus after heat stroke. Shock 2019; 51: 780-6.

13. Chen F, Li H, Zhu G, Chen X, Tang Z. Sodium tanshinone IIA sulfonate improves inflammation, aortic endothelial cell apoptosis, disseminated intravascular coagulation and multiple organ damage in a rat heat stroke model. Mol Med Rep 2017; 16: 87-94.

14. Tsai YC, Lam KK, Peng YJ, et al. Heat shock protein 70 and AMP-activated protein kinase contribute to 17-DMAG-dependent protection against heat stroke. J Cell Mol Med 2016; 20: 1889-97.

15. Biedenkapp JC, Leon LR. Increased cytokine and chemokine gene expression in the CNS of mice during heat stroke recovery. Am J Physiol Regul Integr Comp Physiol 2013; 305: R978-986.

16. Audet GN, Dineen SM, Quinn CM, Leon LR. Altered hypothalamic inflammatory gene expression correlates with heat stroke severity in a conscious rodent model. Brain Res 2016; 1637: 81-90.

17. Groves A, Kihara Y, Jonnalagadda D, et al. A functionally defined in vivo astrocyte population identified by c-Fos activation in a mouse model of multiple sclerosis modulated by S1P signaling: immediate-early astrocytes (ieAstrocytes). eNeuro 2018; 5: ENEURO.0239-18.2018.

18. Srinivasan R, Lu TY, Chai H, et al. New transgenic mouse lines for selectively targeting astrocytes and studying calcium signals in astrocyte processes in situ and in vivo. Neuron 2016; 92: 1181-95.

19. Garwood CJ, Ratcliffe LE, Simpson JE, Heath PR, Ince PG, Wharton SB. Review: astrocytes in Alzheimer's disease and other age-associated dementias: a supporting player with a central role. Neuropathol Appl Neurobiol 2017; 43: 281-98

20. He X, Liu Y, Lin X, et al. Netrin-1 attenuates brain injury after middle cerebral artery occlusion via downregulation of astrocyte activation in mice. J Neuroinflammation 2018; 15: 268.

21. von Bartheld CS, Bahney J, Herculano-Houzel S. The search for true numbers of neurons and glial cells in the human brain: a review of 150 years of cell counting. J Comp Neurol 2016; 524: 3865-95.

22. Shandra O, Robel S. Imaging and manipulating astrocyte function in vivo in the context of CNS injury. Methods Mol Biol 2019; 1938: 233-46.

23. Jha MK, Jo M, Kim JH, Suk K. Microglia-astrocyte crosstalk: an intimate molecular conversation. Neuroscientist 2019; 25: 227-40. 